\title{
ON THE TURBULENT PRANDTL NUMBER IN STABLY STRATIFIED TURBULENCE BY SECOND ORDER MODELS
}

\author{
S. Naifer ${ }^{1, *}$, M. Bouzaiane $e^{1,2}$
}

\begin{abstract}
The aim of this work is to investigate the behavior of the Turbulent Prandtl number by second order modeling of a stably stratified homogeneous sheared turbulence. By analytic solutions, we have confirmed the asymptotic equilibrium behavior of the turbulent Prandtl number. Then two between the most second order models of turbulence; the Classic Launder-Reece-Model and the sophisticated Craft Launder model are retained. A non dimensional form of transport equations have been obtained when non dimensional parameters are introduced to substitute second order moments. A numerical integration using the fourth order Runge kutta method has been conducted for different values of the gradient Richardson number $\mathrm{R}_{\mathrm{i}}$. In comparison with direct numerical simulation result's of Shih et al. the obtained results by the Craft Launder model has shown for the turbulent Prandtl number the best agreement at moderate values of gradient Richardson number $0.15<\mathrm{R}_{\mathrm{i}}<0.28$. The classic model has shown a great default for the different values of $R_{i}$. No any concordance with retained results of DNS has been obtained by this model. We show also that prediction of this model can be improved by introducing variation and optimization of model constants.
\end{abstract}

\section{Keywords: Prandtl Number, Stably Stratified Turbulence, Second Order Modeling}

\section{INTRODUCTION}

The problem of turbulent mixing in a stably stratified turbulence is a problem of often importance since stably stratified are present in the atmospheric boundary layer, oceans, lakes and many engineering flows. Homogenous turbulence in stratified shear flow has been investigated essentially through direct numerical simulations by several authors during the last three decades. Gerz et al. [1] and Holt et al. [2] have analyzed the behavior of a stratified homogeneous sheared turbulence as a function of the important parameter $R_{i}$. The gradient Richardson number $R_{i}$ indicates the importance of stratification effect to shear effect.Shih et al. [3-4] have accorded much attention to the turbulent Prandtl number than the other works and their results are retained in this work. This number is a widely used parameter in stably stratified homogeneous turbulence.

More recently, Subhas and Derek [5] have argued these results and derived a new relationship for the turbulent Prandtl number in terms of the gradient Richardson number $R_{i}$, and between the turbulent kinetic energy and scalar variance. The second order modeling of stratified homogeneous turbulence remains to our sense an important approach of studying homogeneous turbulence since its application to the great number of engineering flows and industrial applications [6-7]. In a previous work, we are interested to the prediction of equilibrium states in stratified homogenous turbulence with vertical or inclined shear [8-9]. We were focused essentially to the asymptotic behavior of dimensionless kinematic parameters such as the component of the anisotropic tensor and the non dimensional shear number $K S / \varepsilon$.

However to our best knowledge not previous results have been dedicated to the prediction of the turbulent Prandtl number behavior using second order models. This simple and important goal constitutes the motivation of this paper which is organized as follows. In section 2 we present the mathematical formulation of a stratified homogeneous turbulence by the transport equation of second order moments of turbulence. Some analytical comments are than proposed for the turbulent Prandtl number $\operatorname{Pr}_{t}$ and the ratio $L_{M} / L_{E}$. In section 3 the second order modeling of transport equations is described and the retained second order models of turbulence are introduced. A non dimensional form of the transport equation is also obtained, parameterized by

This paper was recommended for publication in revised form by Regional Editor Jaaf Hoffman Hoffman

${ }^{1}$ Department of Physics, Faculty of Science of Tunis ,2092 El Manar 2, Tunis, Tunisia

${ }^{2}$ Department of Physics, Faculty of Sciences of Bizerte, Zarzouna, Banzart 7021, Tunisia

*E-mail address: saida17naifer@gmail.com, mbouzaiane@yahoo.fr

Orcid id: https://orcid.org/0000-0002-2111-9460

Manuscript Received 15 March 2018, Accepted 7 May 2018 
the gradient Richardson number $R_{i}$. In section 4 the numerical integrations conducted and the principal results are discussed. The section 5 the principal concluding of this work is dressed.

\section{MATHEMATICAL FORMULATION GENERAL EQUATIONS}

In the case of a stably stratified homogenous sheared turbulence, the basic transport equation for the components $\overline{u_{i} u_{j}}$ of the Reynolds stress, the turbulent kinetic energy, the turbulent scalar flux and scalar variance may be obtained by standard method [2]:

$$
\begin{gathered}
\frac{d \overline{d t} \overline{u_{i} u_{j}}=P_{i j}-B_{i j}+\Phi_{i j}-\varepsilon_{i j}}{\frac{d K}{d t}=P-B-\varepsilon} \\
\frac{d}{d t}\left(\overline{u_{i} \theta}\right)=P_{i \theta}-B_{i \theta}+\Phi_{i \theta}-\varepsilon_{i \theta} \\
\frac{d}{\theta^{2}}=P_{\theta}-2 \varepsilon_{\theta}
\end{gathered}
$$

In these equations terms noted $P$ are production terms due to mean velocity and mean scalar gradients:

$$
\begin{gathered}
P_{i j}=-\bar{u}_{i} u_{k} \bar{U}_{k, j}-\bar{u}_{j} u_{k} \bar{U}_{k, i} \\
P=-{\overline{u_{i} u_{k}}}_{\bar{U}}{ }_{k, i} \\
P_{i \theta}=-\overline{u_{i} u_{k}} S_{T}-\overline{u_{j} \theta} \bar{U}_{i, k} \\
P_{\theta}=-2 \overline{u_{2} \theta} S_{T}
\end{gathered}
$$

$\Phi_{i j}$ and $\Phi_{i \theta}$ are the pressure strain correlation and pressure scalar gradient correlation terms:

$$
\begin{gathered}
\Phi_{i j}=\left(\overline{p u_{i, j}+p u_{j, i}}\right) \\
\Phi_{i \theta}=\frac{1}{\rho_{0}} \overline{p \theta_{, i}}
\end{gathered}
$$

$B_{i j}$ and $B$ are buoyancy terms:

$$
\begin{gathered}
B_{i j}=\frac{g}{\rho_{0}}\left(\overline{u_{i} \theta} \delta_{j 2}+\overline{u_{j} \theta} \delta_{i 2}\right) \\
B=\frac{g}{\rho_{0}} \overline{u_{i} \theta} \delta_{i 2}
\end{gathered}
$$




$$
B_{i \theta}=\frac{g}{\rho_{0}} \overline{\theta^{2}} \delta_{i 2}
$$

and $\varepsilon$ are dissipation due to molecular effects terms:

$$
\begin{gathered}
\varepsilon_{i j}=2 v \overline{\frac{\partial u_{i}^{\prime} \partial u_{j}^{\prime}}{\partial x_{k} \partial x_{k}}} \\
\varepsilon=\varepsilon_{i i} / 2=2 v \overline{\frac{\partial u_{i}^{\prime} \partial u_{i}^{\prime}}{\partial x_{k} \partial x_{k}}} \\
\varepsilon_{i \theta}=(\alpha+\gamma) \overline{u_{i, k} \theta_{, k}} \\
\varepsilon_{\theta}=2 \alpha \overline{\frac{\partial \theta}{\partial x_{k}} \frac{\partial \theta}{\partial x_{k}}}
\end{gathered}
$$

Now, analytical comments are developed for transport equations (1) - (4). The behavior of the turbulent Prandtl number $P r_{t}$ and the ratio $L_{M} / L_{E}$ will be investigated. In their direct numerical simulations, Holt et al. [2] have confirmed that the non linear and viscosity effects are very small comparing to productions terms at high shear $(\tau \gg)$ ). If we take into account of this hypothesis in the transport equations (1) - (4), a linear set of differential equations are obtained and written in the following form:

$$
\begin{gathered}
\frac{d}{d t} \overline{u_{1}^{2}}=-2 S \overline{u_{1} u_{2}} \\
\frac{d}{d t} \overline{u_{2}^{2}}=2 \beta g \overline{u_{2} \theta} \\
\frac{d}{d t} \overline{u_{3}^{2}}=O \\
\frac{d}{d t} \overline{u_{1} u_{2}}=-S \overline{u_{2}^{2}}+\beta g \overline{u_{1} \theta} \\
\frac{d}{d t} \overline{u_{1} \theta}=-S_{T} \overline{u_{1} u_{2}}-S \overline{u_{2} \theta} \\
\frac{d}{d t} \overline{u_{2} \theta}=-S_{T} \overline{u_{2}^{2}}+\beta g \overline{\theta^{2}} \\
\frac{d}{d t} \overline{\theta^{2}}=-2 S_{T} \overline{u_{2} \theta}
\end{gathered}
$$


The Laplace transform is retained for integrating the linear differential system, the principal solutions are written as follows:

$$
\begin{gathered}
\overline{u_{1} u_{2}}=A_{12} \operatorname{Ch}\left(\sqrt{R_{i}} \tau\right)+B_{12} S h\left(\sqrt{R}_{i} \tau\right)+D_{12} \operatorname{Ch}\left(2 \sqrt{R_{i}} \tau\right) \\
+E_{12} \operatorname{Sh}\left(2 \sqrt{R_{i}} \tau\right) \\
\overline{u_{2} \theta}=C_{2 \theta} \operatorname{Ch}\left(2 \sqrt{R_{i}} \tau\right)-B_{2 \theta} \operatorname{Sh}\left(2 \sqrt{R_{i}} \tau\right) \\
2 K=\overline{q^{2}}=\overline{u_{1}^{2}}+\overline{u_{2}^{2}}+\overline{u_{3}^{2}} \\
\overline{q^{2}=2 k}=A_{q} \operatorname{Ch}\left(\sqrt{R_{i}} \tau\right)+B_{q} \operatorname{Sh}\left(\sqrt{R_{i}} \tau\right)+D_{q} C h\left(2 \sqrt{R_{i}} \tau\right) \\
+E_{q} \operatorname{Sh}\left(2 \sqrt{R_{i}} \tau\right)+F_{q} \\
\overline{\theta^{2}}=A_{\theta \theta} \operatorname{Ch}\left(2 \sqrt{R_{i}} \tau\right)+B_{\theta \theta} \operatorname{Sh}\left(2 \sqrt{R_{i}} \tau\right)
\end{gathered}
$$

Where coefficients A, B, D are functions of initial values of turbulent parameters and the gradient Richardson number $\mathrm{R}_{\mathrm{i}}$. These solutions will now be used to study the asymptotic behavior (when $\tau \rightarrow \infty$ ) of the dimensionless parameters: the turbulent Prandtl number $\operatorname{Pr}_{t}$ and the ratio $L_{M} / L_{E}$. Firstly we deduce the expressions of these parameters:

$$
\begin{aligned}
& P_{t}=\frac{S_{T}}{S} \frac{\overline{u_{1} u_{2}}}{\overline{u_{2} \theta}} \\
& =\frac{S_{T}}{S} \frac{A_{12} C h\left(\sqrt{R_{i}} \tau\right)+B_{12} \operatorname{Sh}\left(\sqrt{R_{i}} \tau\right)+D_{12} C h\left(2 \sqrt{R_{i}} \tau\right)+E_{12} \operatorname{Sh}\left(2 \sqrt{R_{i}} \tau\right)}{C_{2 \theta} \operatorname{Ch}\left(2 \sqrt{R_{i} \tau}\right)-B_{2 \theta} \operatorname{Sh}\left(2 \sqrt{R_{i}} \tau\right)} \\
& \frac{L_{M}}{L_{E}}=\frac{S_{T}}{S}\left(\overline{\frac{q^{2}}{\bar{\theta}^{2}}}\right)^{1 / 2} \\
& =\frac{S_{T}}{S}\left(\frac{A_{q} C h\left(\sqrt{R_{i}} \tau\right)+B_{q} \operatorname{Sh}\left(\sqrt{R_{i}} \tau\right)+D_{q} \operatorname{Ch}\left(2 \sqrt{R_{i}} \tau\right)+E_{q} \operatorname{Sh}\left(2 \sqrt{R_{i}} \tau\right)+F_{q}}{A_{\theta \theta} C h\left(2 \sqrt{R_{i}} \tau\right)+B_{\theta \theta} \operatorname{Sh}\left(2 \sqrt{R_{i}} \tau\right)}\right)^{1 / 2}
\end{aligned}
$$

And at high shear $(\tau>>)$, we obtain respectively:

$$
\begin{aligned}
& \left(P r_{t}\right)_{\infty}=\lim _{\tau \rightarrow \infty}\left(\frac{S_{T}}{S} \frac{\overline{u_{1} u_{2}}}{\overline{u_{2} \theta}}\right)=\frac{S_{T}}{S} \frac{D_{12}+E_{12}}{C_{2 \theta}-B_{2 \theta}} \\
& \left(\frac{L_{M}}{L_{E}}\right)_{\infty}=\lim _{\tau \rightarrow \infty}\left(\frac{S_{T}}{S}\left(\frac{\overline{q^{2}}}{\overline{\theta^{2}}}\right)^{1 / 2}\right)=\frac{S_{T}}{S} \frac{\left(D_{q}+E_{q}\right)^{1 / 2}}{\left(A_{\theta \theta}+B_{\theta \theta}\right)^{1 / 2}}
\end{aligned}
$$


We conclude that at high shear $(\tau>>)$, the two dimensionless scalar parameters tend to equilibrium states functions of the initial values of turbulent parameters and the gradient Richardson number $R_{i}$. This analytic solution is important since it confirms that at equilibrium, buoyancy terms equilibrate production terms. It confirms also the result of direct numerical simulation of Shih et al. [4] and the study of Subhas and Derek [5]. This approach is only a qualitative one and a more quantitative study of the evolution of the turbulent Prandtl number $\operatorname{Pr}_{t}$ and the ratio $L_{M} / L_{E}$ will be addressed in the following section when second order modeling of transport equations is retained.

\section{SECOND ORDER MODELING}

At this step of our work and with the aim of obtaining a closed set of equations, the second order modeling is the approach retained here. Second order models are retained for nonlinear terms of pressure strain correlation, pressure scalar gradient correlation and transport equation of dissipation.

In a stratified shear flow, the correlations $\Phi_{i j}$ and $\Phi_{i \theta}$ are classically [11] decomposed on three contributions:

$$
\begin{gathered}
\Phi_{i j}=\Phi_{i j}^{1}+\Phi_{i j}^{2}+\Phi_{i j}^{3} \\
\Phi_{i \theta}=\Phi_{i \theta}^{1}+\Phi_{i \theta}^{2}+\Phi_{i \theta}^{3}
\end{gathered}
$$

Where the contributions 1 are the return-to-the isotropy terms, the terms 2 represent the interaction between mean and turbulent flows. The terms 3 are terms due to buoyancy.

Two between the most known second order models are retained for the pressure strain correlation and the pressure scalar gradient correlation and the evolution equation of the dissipation rate $\varepsilon$. The classic second order model of Launder, Reece and Rodi (LRR) [12] in one hand and the sophisticated model of Craft and Launder (CL) [13] have been retained. For its success in many different applications of stratified homogeneous shear flow [8-10], the Zeman Lumley model is retained for the third contributions of the pressure strain correlation and pressure scalar gradient correlation:

$$
\begin{gathered}
\Phi_{i j}^{3}=C_{3}\left(\frac{1}{\rho_{0}}\left(g_{i} \overline{u_{j} \theta}+g_{j} \overline{u_{i} \theta}-\frac{2}{3} \delta_{i j} \frac{g_{m}}{\rho_{0}} \overline{u_{m} \theta}\right)\right) \\
\Phi_{i \theta}^{3}=C_{\theta 3} \frac{1}{\rho_{0}} g_{i} \overline{\theta^{2}}
\end{gathered}
$$

Where $C_{3}=0.5$ and $C_{\theta 3}=0.5$.

Introducing the non dimensional time $\tau=S t$, a modeled form retained for the transport equations of the mentioned parameters $b_{i j}, S K / \varepsilon, P r_{t}$ and $L_{M} / L_{E}$ are obtained by:

$$
\begin{aligned}
\frac{d}{d \tau} b_{i j}= & -\left(b_{i 2}+\frac{\delta_{i 2}}{3}\right) \delta_{j 1}-\left(b_{j 2}+\frac{\delta_{j 2}}{3}\right) \delta_{i 1}+\frac{\beta g}{2 S K}\left(\overline{u_{i} \theta} \delta_{j 2}+\overline{u_{j} \theta} \delta_{i 2}\right)+\frac{1}{2 S K} \Phi_{i j}-\frac{1}{3} \frac{\varepsilon}{S K} \delta_{i j} \\
& +\left(b_{i j}+\frac{\delta_{i j}}{3}\right)\left(2 b_{12}-\frac{\beta g}{S K} \overline{u_{2} \theta}+\frac{\varepsilon}{S K}\right) \\
\frac{d}{d \tau}\left(\frac{S K}{\varepsilon}\right)= & \left(1+C_{\varepsilon 1}-C_{\varepsilon 3}\right)\left(\frac{S K}{\varepsilon}\right) \frac{2 R_{i} b_{12}}{P r_{t}}+\left(C_{\varepsilon 1}-1\right) 2 b_{12}\left(\frac{S K}{\varepsilon}\right)-1+C_{\varepsilon 2}
\end{aligned}
$$




$$
\begin{aligned}
& \frac{d}{d \tau} P r_{t}= {\left[-\left(b_{22}+1 / 3\right)+\frac{\Phi_{12}}{2 K S}\right] \frac{P r_{t}}{b_{12}}+R_{i} \frac{\overline{\theta u_{1}}}{\overline{\theta u_{2}}}+\left[b_{22}+\frac{1}{3}-\frac{R_{i}}{\left(L_{M} / L_{E}\right)^{2}}-\frac{\Phi_{2 \theta}}{2 K S_{T}}\right] \frac{\left(P r_{t}\right)^{2}}{b_{12}} } \\
& \frac{d}{d \tau}\left(\frac{L_{M}}{L_{E}}\right)=\left(R_{i} P r_{t}-1\right) b_{12}\left(\frac{L_{M}}{L_{E}}\right)+\left(\frac{1}{r}-\left(\frac{K}{2}\right)^{1 / 2}\right)\left(\frac{\varepsilon}{S K}\right)\left(\frac{L_{M}}{L_{E}}\right)+\frac{b_{12}}{P r_{t}}\left(\frac{L_{M}}{L_{E}}\right)^{3} \\
& \frac{d}{d \tau}\left(\frac{\overline{\theta u_{1}}}{\overline{\theta u_{2}}}\right)=\left(\frac{\Phi_{1 \theta}}{2 K S_{T} b_{12}}-1\right) P r_{t}+\left(\frac{\overline{\theta u_{1}}}{\overline{\theta u_{2}}}\right)\left[b_{22}+\frac{1}{3}-\frac{R_{i}}{\left(L_{M} / L_{E}\right)^{2}}-\frac{\Phi_{2 \theta}}{2 K S_{T}}\right] \frac{P r_{t}}{b_{12}}
\end{aligned}
$$

At this step, a numerical integration of the differential equations is started. Obtained results will be discussed in the following sections.

\section{NUMERICAL INTEGRATION AND RESULTS}

Two non linear systems of seven differential equations are obtained corresponding to the two retained models. The numerical integration is advanced in time using the fourth order Runge-Kutta method. Numerical integration has been advanced to long time evolution $\tau=12$. The principal results are now discussed : A plot of the turbulent Prandtl number $P r_{t}$ for weak stratification, $\mathrm{R}_{\mathrm{i}}=0.05,0.06$ and 0.1, is shown in Figures 1. The Craft Launder model shows an overestimation of about $50 \%$ direct numerical simulations values. The LRR model indicates an under estimation of these values.

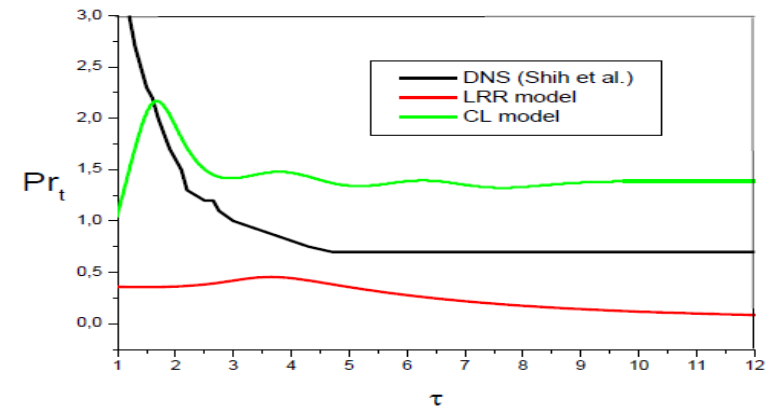

a. for $R_{i}=0.05$

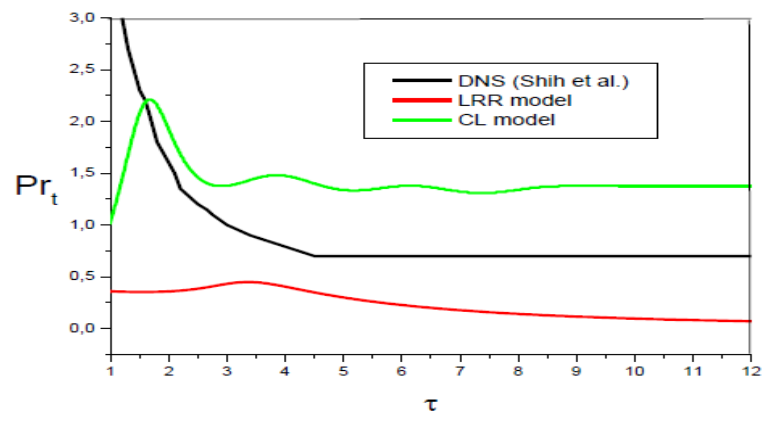

b. for $R_{i}=0.06$

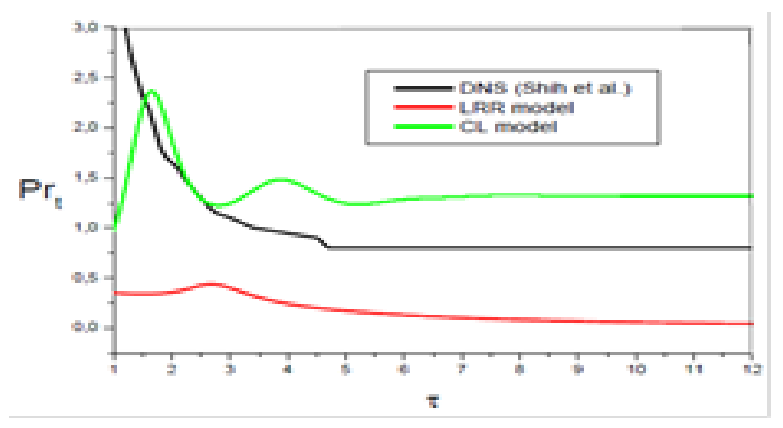

c. for $R_{i}=0.1$

Figure 1. Time evolution of $\mathrm{Pr}_{t}$ for Data of Shih et al. and LRR and CL models

The estimation of the CL model becomes better for moderate values of $R_{i}$ equals respectively to 0.15 , 0.18 and 0.25 . The differences between predictions of CL model and direct numerical simulations values is less important than in the case of weak values of $\mathrm{R}_{\mathrm{i}}$ as indicated in Figures 2.An over estimation of $10 \%$ the direct numerical values for $\mathrm{R}_{\mathrm{i}}=0.18$ and an under estimation of $10 \%$ values of direct numerical simulation for $\mathrm{R}_{\mathrm{i}}=0.25$ has been observed by CL model. The LRR model does not indicate interesting behavior for the moderated or 
weak stratification for the turbulent Prandtl number. A great difference between LRR model predictions and the values of the direct numerical simulation is observed for both weak and moderate stratification.

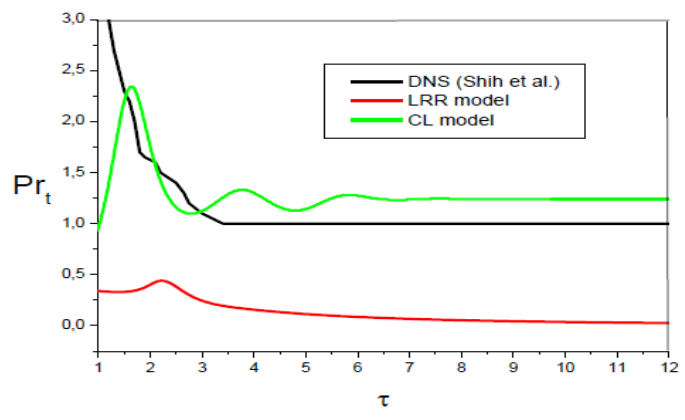

a. for $R_{i}=0.15$

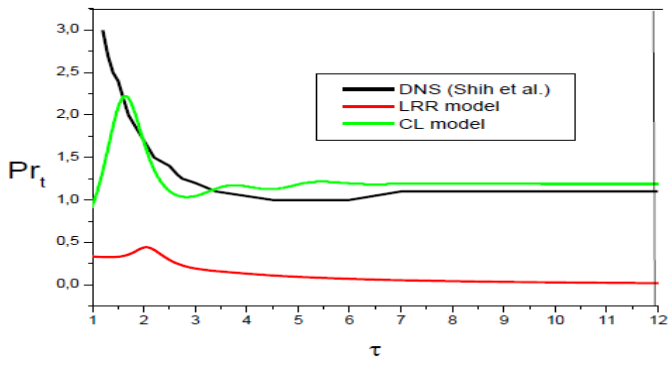

b. for $R_{i}=0.18$

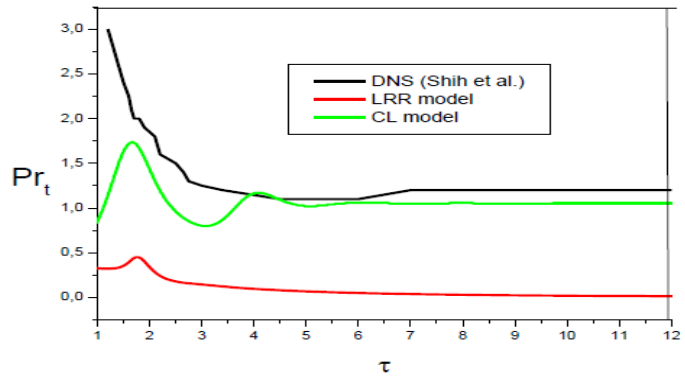

c. for $R_{i}=0.25$

Figure 2. Time evolution of $P r_{t}$ for Data of Shih et al. and LRR and CL models

Figures 3 indicate plot of Prandtl number at high values of the gradient Richardson number $R_{i}=0.37$, 0.4 and 0.6. Here no concordance between models predictions and values of direct numerical simulations has been observed. An under estimation of $50 \%$ values of DNS of Shih et al. has been observed for $\mathrm{R}_{\mathrm{i}}=0.37$ and $\mathrm{R}_{\mathrm{i}}=0.4$ by Craft Launder model whereas no any concordance has been observed between the LRR model and values of direct numerical simulations of Shih et al. [4].

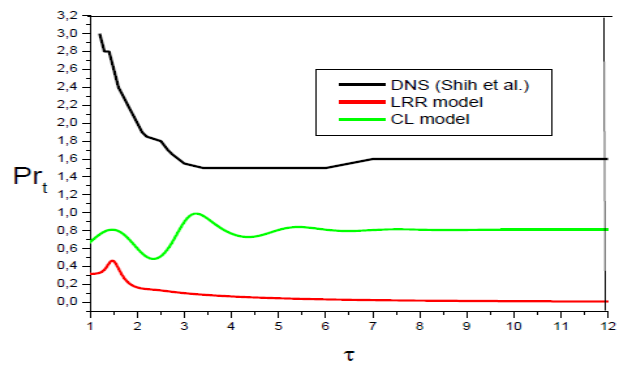

a. for $R_{i}=0.37$

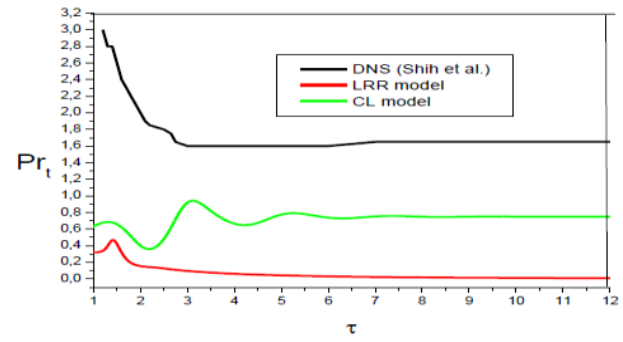

b. for $R_{i}=0.4$

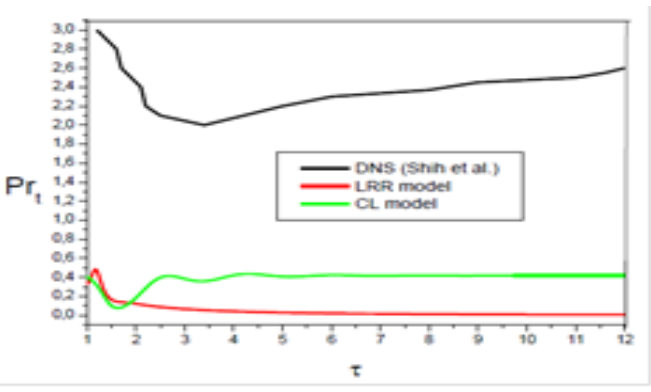

c. for $R_{i}=0.6$

Figure 3. Time evolution of $\mathrm{Pr}_{t}$ for Data of Shih et al. and LRR and CL models 
Table 1 indicates equilibrium values of turbulent Prandtl number by models and direct numerical simulations. Here also acceptable concordance with values of DNS is obtained for moderate values of the gradient Richardson number $R_{i}$ by the Craft Launder model. An over estimation and an underestimation is obtained by this model for weak and high stratification respectively.

Table 1. Equilibrium values of $P r_{t}$ for different values of $R_{i}$

\begin{tabular}{|c|c|c|c|}
\hline $\mathbf{R}_{\mathbf{i}}$ & $\begin{array}{c}\left(\mathrm{Pr}_{t}\right)_{\infty} \\
(\mathbf{D N S} \\
\text { (Shih et al.)) }\end{array}$ & $\begin{array}{c}\left(\mathrm{Pr}_{t}\right)_{\infty} \\
(\mathbf{L R R} \\
\text { Model })\end{array}$ & $\begin{array}{c}\left(\mathrm{Pr}_{t}\right)_{\infty} \\
(\mathbf{C L} \\
\text { Model })\end{array}$ \\
\hline 0.05 & 0.7 & 0 & 1.39 \\
\hline 0.06 & 0.7 & 0 & 1.38 \\
\hline 0.1 & 0.8 & 0 & 1.32 \\
\hline 0.15 & 1 & 0 & 1.24 \\
\hline 0.18 & 1.05 & 0 & 1.19 \\
\hline 0.25 & 1.25 & 0 & 1.05 \\
\hline 0.37 & 1.6 & 0 & 0.81 \\
\hline 0.4 & 1.7 & 0 & 0.75 \\
\hline 0.6 & - & 0 & 0.418 \\
\hline
\end{tabular}

To improve performance of the LRR model, correction to model coefficient is retained by many authors. The first coefficient $C_{1}$ of the return to isotropy model of the pressure strain correlation $\Phi_{i j}$ is generally the coefficient concerned in this modification. Here, we are interested to modify this coefficient $C_{1}$ with small variations around the known value $C_{1}=-1.8$. The equilibrium values of $\operatorname{Pr}_{t}$ obtained as function of $C_{1}$ are presented on the following table:

Table 2. Equilibrium values of $P r_{t}$ for different values of $C_{1}$ for $R_{i}=0.05$ and $R_{i}=0.37$

\begin{tabular}{|c|c|c|}
\cline { 2 - 3 } \multicolumn{1}{c|}{} & \multicolumn{2}{c|}{$\left(\operatorname{Pr}_{t}\right)_{\infty}$} \\
\cline { 2 - 3 } & $R_{i}=0.05$ & $R_{i}=0.37$ \\
\hline LRR $C_{1}=-0.35$ & 0.504 & 0.137 \\
\hline LRR $C_{1}=-0.4$ & 0.423 & 0.109 \\
\hline LRR $C_{1}=-0.5$ & 0.37 & 0 \\
\hline LRR $C_{1}=-1$ & 0.177 & 0 \\
\hline LRR $C_{1}=-1.8$ & 0 & 0 \\
\hline LRR $C_{1}=-2$ & 0 & 0 \\
\hline DNS & 0.7 & 1.6 \\
\hline
\end{tabular}

Another optimization which we propose here is for the coefficient $C_{\theta 1}$ of the return to isotropy of the model of pressure scalar gradient correlation. We obtain the following table of equilibrium values of $\operatorname{Pr}_{t}$ for different values of this coefficient:

We see that the equilibrium value of $P r_{t}$ is very sensitive to variation of the coefficient $C_{1}$ for the weak value 0.05 of the gradient Richardson number $R_{i}$. When $C_{1}$ equal -0.5 and -1 , a better approximation of the DNS value is observed by LRR model. The best approximation is observed for $C_{1}=-0.5$. For $R_{i}=0.37$ no improve of the LRR model has been observed. The same observation is also available for variation of the coefficient $C_{\theta 1}$ of the return to isotropy model of pressure scalar gradient correlation. Variation of model coefficient can improve prediction of LRR model only for weak values of $R_{i}$. 
Table 3. Equilibrium values of $P r_{t}$ for different values of $C_{\theta 1}$ for $R_{i}=0.05$ and $R_{i}=0.37$

\begin{tabular}{|c|c|c|}
\cline { 2 - 3 } \multicolumn{1}{c|}{} & \multicolumn{2}{c|}{$\left(P r_{t}\right)_{\infty}$} \\
\cline { 2 - 3 } \multicolumn{1}{c|}{} & $R_{i}=0.05$ & $R_{i}=0.37$ \\
\hline LRR $C_{\theta 1}=-8$ & 0.673 & 0 \\
\hline LRR $C_{\theta 1}=-6$ & 0.338 & 0 \\
\hline LRR $C_{\theta 1}=-3.6$ & 0.102 & 0 \\
\hline LRR $C_{\theta 1}=-3.2$ & 0 & 0 \\
\hline DNS & 0 & 0 \\
\hline
\end{tabular}

It is essentially here to precise that coefficients $C_{1}$ and $C_{\theta 1}$ of return to isotropy terms are generally the only coefficients which we can modify. The values of the others coefficients of the linear terms of pressure strain correlation and pressure scalar gradient correlations are exact. They cannot be modified since they are submitted in addition to kinematic constraints (continuity and symmetry...) to the strong form of realisability condition [13].

Figures 4 show the plot of the ratio $L_{M} / L_{E}$. No concordance has been observed for the case of weak stratification between predictions models and the direct numerical simulation values. An acceptable agreement between predictions models and DNS at high values of the gradient Richardson number $R_{i}=0.37,0.4$ and 0.6. More precisely, for different values of gradient Richardson number $R_{i}$, the ratio $L_{M} / L_{E}$ reaches a constant value from $\tau$ greater than 6 . For the Craft Launder model, we observe in table 4 an over estimation of asymptotic values of about $50 \%$ for the values $R_{i}=0.37$ and 0.4 . An excellent agreement with the value of DNS of Shih et al. has been observed for the value 0.6 of the gradient Richardson number.

The default of the LRR model is here clear also no concordance has been observed between LRR model and DNS values.

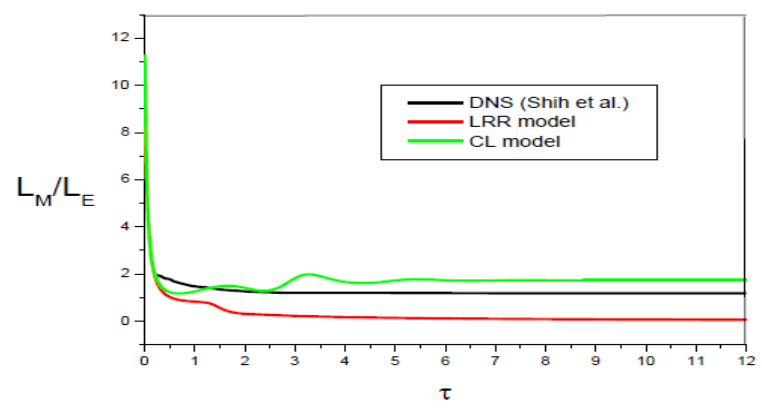

a. for $R_{i}=0.37$

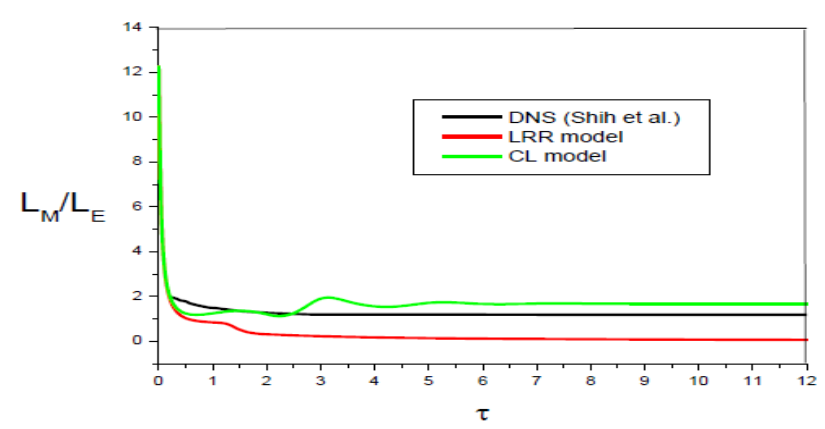

b. for $R_{i}=0.4$

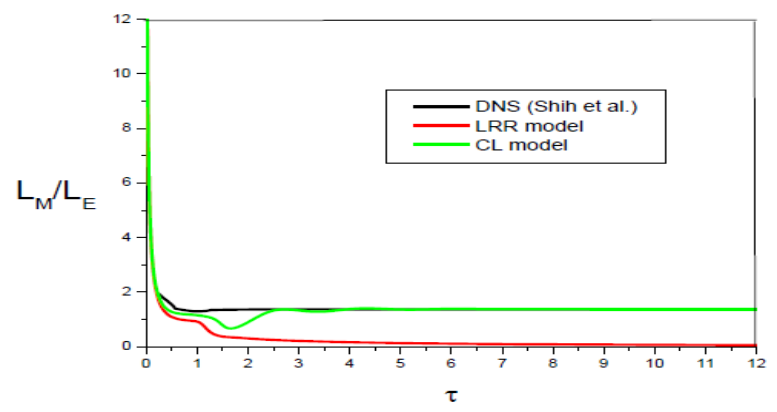

c. for $R_{i}=0.6$

Figure 4. Time evolution of $L_{M} / L_{E}$ for Data of Shih et al. and LRR and CL models 
Table 4. Equilibrium values of $L_{M} / L_{E}$ for different values of $R_{i}$

\begin{tabular}{|c|c|c|c|}
\hline $\mathbf{R}_{\mathbf{i}}$ & $\begin{array}{c}\left(L_{M} / L_{E}\right)_{\infty} \\
\text { (DNS (Shih } \\
\text { et al.) })\end{array}$ & $\begin{array}{c}\left(L_{M} / L_{E}\right)_{\infty} \\
\text { (LRR } \\
\text { model) }\end{array}$ & $\begin{array}{c}\left(L_{M} / L_{E}\right)_{\infty} \\
(\mathbf{C L} \\
\text { model) }\end{array}$ \\
\hline 0.37 & 1.12 & 0 & 1.73 \\
\hline 0.4 & 1.14 & 0 & 1.68 \\
\hline 0.6 & 1.36 & 0 & 1.38 \\
\hline
\end{tabular}

\section{CONCLUDING REMARKS}

In this work, we are interested to the study of the evolution of scalar parameters in stably stratified homogeneous sheared turbulence using second order models of turbulence. The turbulent Prandtl number is the principal parameter concerned. The ratio $L_{M} / L_{E}$ of mechanical length scale to scalar length scale is also investigated. At a first step, we have confirmed by analytical linear solutions, deduced from Laplace transform and available only at high shear that the two mentioned dimensionless parameters have a general tendency to asymptotic equilibrium states, observed by the DNS of Shih et al. In the second step, two second order models have been retained for pressure strain correlation and pressure scalar gradient correlation to model transport equations. The fourth order Runge-Kutta method has been retained for integrating two non linear systems of seven differential equations.

The obtained results have shown an acceptable concordance between the Craft Launder model and DNS values at moderate values of the gradient Richardson number 0.15, 0.18 and 0.25 . A great default of the LRR model has been observed here with the values of the DNS of Shih et al. It confirms the default of this model in stratified homogeneous turbulence observed in our previous work [8]. To improve its prediction, we make variations of the coefficients $C_{1}$ and $C_{\theta 1}$ of the terms of return to isotropy of pressure strain correlation and pressure scalar gradient correlation. Only at weak value of the gradient Richardson number $R_{i}=0.05$ a better approximation of the DNS values of Shih et al. [4] has been observed. For the moderate value of gradient Richardson number $R_{i}=0.37$ no improve of the LRR model has been observed for variations of coefficients $C_{1}$ and $C_{\theta 1}$. To improve predictions of LRR model and CL model, at high gradient Richardson number, we think that introduction of correction function by analogy as that introduced by Speziale et al. [14] and Younis et al. [15] in the case of turbulence, with rotation can make an important direction of investigation in a future work.

\section{NOMENCLATURE}

$\begin{array}{ll}b_{i j}=\left(\overline{u_{i} u_{j}} / 2 K\right)-\left(\delta_{i j} / 3\right) & \text { Reynolds stress anisotropy tensor } \\ \mathrm{g}_{\mathrm{i}} & \text { Gravitational acceleration }\left[\mathrm{m} / \mathrm{s}^{2}\right] \\ \mathrm{K} & \text { Turbulent kinetic energy }\left[\mathrm{m}^{2} / \mathrm{s}^{2}\right] \\ L_{M}=(2 K)^{1 / 2} / S & \text { Mechanical length scale }[\mathrm{m}] \\ L_{E}=\left(\overline{\theta^{2}}\right)^{1 / 2} / S_{T} & \text { Scalar length scale }[\mathrm{m}] \\ \mathrm{P} & \\ \mathrm{p} & \text { Production due to mean kinematic and scalar gradients } \\ p & \left.\text { Pressure [N/m }{ }^{2}\right] \\ P r_{t} & \text { Fluctuation of the pressure }\left[\mathrm{N} / \mathrm{m}^{2}\right] \\ R_{i}=g \beta S_{T} / S^{2} & \text { Turbulent Prandtl number } \\ \mathrm{S} & \text { Dimensionless Richardson number } \\ S_{i j} & \text { Shear rate }\left[\mathrm{s}^{-1}\right] \\ \mathrm{S}_{\mathrm{T}} & \text { The mean strain rate tensor }\left[\mathrm{s}^{-1}\right] \\ \mathrm{t} & \text { Mean scalar gradient }\left[{ }^{\circ} \mathrm{C} / \mathrm{m}\right] \\ \mathrm{T} & \text { Time [s] } \\ & \text { Temperature }\left[{ }^{\circ} \mathrm{C}\right]\end{array}$




\begin{tabular}{ll}
$u_{i}$ & i-th component of the fluctuating velocity $[\mathrm{m} / \mathrm{s}]$ \\
$\overline{U_{i}}$ & i-th component of mean velocity $[\mathrm{m} / \mathrm{s}]$ \\
$\overline{u_{i} u_{j}}$ & Reynolds stress tensor $\left[\mathrm{m}^{2} / \mathrm{s}^{2}\right]$ \\
$\overline{u_{i} \theta}$ & Turbulent scalar flux $\left[\mathrm{m}^{\circ} \mathrm{C} / \mathrm{s}\right]$ \\
$\mathrm{W}_{\mathrm{ij}}$ & Mean vorticity tensor $\left[\mathrm{s}^{-1}\right]$ \\
$\mathrm{x}_{\mathrm{i}}$ & Component of an orthonormal Cartesian coordinate system $[\mathrm{m}]$ \\
$\mathrm{Greek} \mathrm{Symbols}$ & \\
$\alpha$ & Thermal diffusivity of fluid $\left[\mathrm{m}^{2} / \mathrm{s}\right]$ \\
$\beta$ & Thermal expansion coefficient $\left[\mathrm{K}^{-2}\right]$ \\
$\delta_{\mathrm{ij}}$ & Kronecker Symbol \\
$\varepsilon$ & Dissipation due to molecular effects $\left[\mathrm{m}^{2} / \mathrm{s}^{3}\right]$ \\
$\tau$ & Non dimensional time $(\tau=S t)$ \\
$\theta$ & Fluctuation of the scalar $\left[{ }^{\circ} \mathrm{C}\right]$ \\
\hline$\theta^{2}$ & Temperature variance $\left[{ }^{\circ} \mathrm{C}^{2}\right]$ \\
$\mu$ & Dynamic viscosity $\left[\mathrm{kg} \cdot \mathrm{m}^{-1} \cdot \mathrm{s}^{-1}\right]$ \\
$v$ & Kinematic viscosity $\left[\mathrm{m}^{2} / \mathrm{s}\right]$ \\
$\rho_{0}$ & Reference density of the fluid $\left[\mathrm{kg} / \mathrm{m}^{3}\right]$ \\
$\Phi_{\mathrm{ij}}$ & Pressure-strain correlation $\left[\mathrm{m}^{2} / \mathrm{s}^{3}\right]$ \\
& Pressure-temperature gradient correlation $\left[{ }^{\circ} \mathrm{C} . \mathrm{m} / \mathrm{s}^{2}\right]$ \\
&
\end{tabular}

\section{REFERENCES}

[1] Gerz, T., Shumann, U., Elghobachi, S. Direct numerical simulation of stratified homogeneous turbulent shear flow. Journal of Fluid Mechanics, 1989 ; 200, 563-594. https://doi.org/10.1017/S0022112089000765.

[2] Holt, S. F., Koseff, J. R., Ferziger, J. H. A numerical study of the evolution and structure of homogeneous stably stratified sheared turbulence. Journal of Fluid Mechanic, 1992; 237, 499-539. https://doi.org/10.1017/S0022112092003513.

[3] Shih, L. H., Koseff, J. R., Ferziger, J. H., and Rehmann, C. R. Scaling and parameterization of stratified homogeneous turbulent shear flow. Journal of Fluid Mechanics, 2000; 412, 1-20. https://doi.org/10.1017/S0022112000008405.

[4] Shih, L. H., Koseff, J. R., Ivey, G. N., and Ferziger, J. H. Parameterization of turbulent fluxes and scales using homogeneous sheared stably stratified turbulence simulations. Journal of Fluid Mechanics, 2005; 525, 193-214. https://doi.org/10.1017/S0022112004002587.

[5] Subhas, K. Venayagamoorthy and Derek, D. Stretch. On the turbulent Prandtl number in homogeneous stably stratified turbulence. Journal of Fluid Mechanics, 2010; 644, 359-369. https://doi.org/10.1017/S002211200999293X.

[6] Fatima MADI AROUS. Numerical simulation with a Reynolds stress turbulence model of flow and heat transfer in rectangular cavities with different aspect ratios. Journal of Thermal Science and Technology, 2016; 11(1), 1-13. doi: 10.1299/jtst.2016jtst0012.

[7] Abay, K., Colak, U., Yüksek, L. Computational fluid dynamics analysis of flow and combustion of a diesel engine. Journal of Thermal Engineering, 2018; 4(2), 1878-1895. doi: 10.18186/journal-of-thermalengineering.388333.

[8] Bouzaiane, M., Ben Abdallah, H., and Lili, T. A study of the asymptotic behaviour of dimensionless parameters in stably stratified sheared turbulence. Journal of Turbulence, 2003; 4, N2. http://dx.doi.org/10.1088/1468-5248/4/1/002.

[9] Bouzaiane, M., Ben Abdallah, H., and Lili, T. A second-order modelling of a stably stratified sheared turbulence submitted to a non-vertical shear. Journal of Turbulence, 2004; 5, N33. http://dx.doi.org/10.1088/1468-5248/5/1/033. 
[10] Thamri, N. L., Bouzaiane, M., Lili, T. A study of equilibrium states of homogeneous turbulence submitted to an inclined shear. International Journal of Computer Science Engineering, 2012; 1(2), 126-139.

[11] Launder, B. E. On the effects of a gravitational field on the turbulent transport of heat and momentum. Journal of Fluid Mechanics, 1975; 67, 569-581. https://doi.org/10.1017/S002211207500047X.

[12] Launder, B. E., Reece, G., and Rodi, W. Progress in the development of a Reynolds stress closure. Journal of Fluid Mechanic, 1975; 68, 537-576. https://doi.org/10.1017/S0022112075001814.

[13] Craft, T. J., Launder, B. E. A new model for the pressure/scalar-gradient correlation and its application to homogeneous and inhomogeneous free shear flows. Seventh Symposium on Turbulent Shear Flows, Stanford, California, 1989; 2, 17.1.1-17.1.6.

[14] Speziale, C. G., Sarkar, S., and Gatski, T. B. Modelling the pressure strain correlation of turbulence: an invariant dynamical systems approach. Journal of Fluid Mechanic, 1991; 227, $245-272$. https://doi.org/10.1017/S0022112091000101.

[15] Younis, B. A., Speziale, C. G., Berger, S. A. Accounting for effects of a system rotation on the pressurestrain correlation. AIAA Journal, 1998; 36(9), 1746-1748. doi: 10.2514/3.14037.

[16] Yuji Kitamura, Akihiro Hori and Toshimasa Yagi. Flux Richardson number and turbulent Prandtl number in adeveloping stable boundary layer. Journal of the Meteorological society of Japan, 2003; 91, 655-666. doi: 10.2151/jmsj.2013-507. 